\title{
TOXICIDAD DE NANOPARTÍCULAS INORGÁNICAS SOBRE LOS MICROORGANISMOS DEL SUELO CON IMPORTANCIA AGRÍCOLA. UNA REVISIÓN
}

\section{TOXICITY OF INORGANIC NANOPARTICLES ON SOIL MICROORGANISMS WITH AGRICULTURAL IMPORTANCE. A REVIEW}

\author{
Sixta L. Palencia' ${ }^{1}$ Enrique M. Combatt ${ }^{2}$, Manuel S. Palencia ${ }^{3}$ \\ Recibido para publicación: Mayo 16 de 2013 - Aceptado para publicación: Noviembre19 2013
}

\begin{abstract}
RESUMEN
El continuo avance de la nanotecnología así como sus aplicaciones en el mejoramiento de la agricultura son una realidad; sin embargo, la información que se posee del impacto de este tipo de tecnología sobre los sistemas biomoleculares es muy escasa. El objetivo del presente documento es construir, desde un punto de vista molecular, un panorama del estado actual de las investigaciones en torno al efecto de las nanopartículas metálicas sobre los microorganismos (MOs) y de este modo contribuir, desde una base teórica, al entendimiento de las posibles interacciones que tienen lugar entre estos materiales y los sistemas biomoleculares de los MOs cuando este tipo de sustancias son liberadas al medioambiente, en particular, en agroecosistemas direccionados a la producción de alimentos. Se concluye que la incertidumbre asociada al efecto de las nanopartículas metálicas (M-NPs) sobre los MOs sigue siendo muy grande, y que en consecuencia, mayores estudios deben ser realizados. En términos generales, las M-NPs pueden interaccionar con biomoléculas mediante diferentes mecanismos que pueden operar de forma simultánea, siendo amplio el espectro de variables que determinan su efecto.
\end{abstract}

Palabras clave: Nanotecnología, nanopartículas metálicas, microorganismos, AgNPs, biomoléculas

\begin{abstract}
The continuous advancement of nanotechnology and its applications in the improving of agriculture are a reality; however, the information about the impact of this technology on biomolecular systems is limited. The aim of this paper is to construct, from a molecular point of view, an overview of the current state of researches

\footnotetext{
`Bacterióloga, Estudiante de Maestría en Microbiología Molecular, Universidad Libre sede Barraquilla, Barranquilla, Colombia ${ }^{2}$ Departamento de Ingeniería Agronómica y Desarrollo Rural, Facultad de Ciencias Agrícolas, Universidad de Córdoba, Montería, Colombia

${ }^{3}$ Departamento de Química, Facultad de Ciencias Naturales y Exactas, Universidad del Valle, Cali, Colombia. Edif. 320 Ofic. 2063 Sede Melendez. (57-2) 3212180, Fax 3212128, manuel.palencia@correounivalle.edu.co
} 
Palencia et al. - Toxicidad de nanopartículas sobre los microorganismos del suelo

around the metal nanoparticles effect on microorganisms (MOs) and thereby to contribute, from a theoretical basis, in the understanding of the possible interactions that take place between these materials and $\mathrm{MO}$ biomolecular systems when such substances are released to the environment, particularly in agroecosystems used for the food production. It is concluded that uncertainty associated with the effect of metal nanoparticles (M-NPs) on MOs is still very wide, and in consequence, further studies should be performed. In general terms, the M-NPs can interact with biomolecules using different mechanisms which can operate simultaneously, with a broad range of variables determining its effect.

Key words: Nanotechnology, metal nanoparticles, microorganisms, AgNPs, biomolecules

\section{INTRODUCCIÓN}

\section{Aspectos fundamentales de la nanotecnología.}

La nanotecnología puede definirse como la tecnología basada en el estudio y la manipulación de la materia en un rango de dimensiones muy pequeño, la nanoescala, la cual en el lenguaje de la nanociencia se define usualmente entre 1 y $100 \mathrm{~nm}$. Para tener una idea de las dimensiones a las que se hace referencia en nanotecnología se debe considerar que, una esfera con un diámetro de 20 nm es mucho más grande que un átomo, una molécula, el ADN o una proteína, sin embargo esa misma esfera es mucho más pequeña que un virus promedio, una bacteria o que el núcleo de una célula humana (Hullmann 2006; Frejo et al. 2011).

La importancia de la nanotecnología radica en que las propiedades químicas y físicas de la materia a nivel nanométrico difieren de las propiedades de la materia a escalas mayores. Así por ejemplo, mientras el grafito, una de las formas alotrópicas del carbono disponible a nivel macro, es de color negro, quebradizo y con una baja conductividad eléctrica; el grafeno es muy flexible, transparente, con alta conductividad eléctrica y térmica. Así, aunque el grafito es un sistema de capas tridimensionales de grafeno, el término grafeno debe ser usado únicamente cuando se discuten las reacciones, relaciones estructurales $u$ otras propiedades de capas individuales (IUPAC 1995). Es importante aclarar que, para que la materia presente estos cambios peculiares en sus propiedades no requiere que como un todo sea inferior a $100 \mathrm{~nm}$, solo basta que al menos en una dimensión se encuentre en el rango de la nanoescala (Hullmann 2006).

En la actualidad las nanopartículas (NPs) se encuentran en muchos productos disponibles en el mercado. Así en protectores solares y cosméticos son usados nanotubos de óxido de titanio $\left(\mathrm{TiO}_{2} \mathrm{NTs}\right)$ y NPs de óxido de zinc (ZnONPs), en detergentes, pinturas, productos fitosanitarios y sistemas antibacterianos se utilizan frecuentemente NPs de plata (AgNPs) y $\mathrm{TiO}_{2}$ NPs (Levard et al. 2012; Park et al. 2012). Las aplicaciones de NPs también incluyen su uso en productos de áreas tan diversas como la electrónica, la medicina, la química, la industria textil, de alimentos, combustibles y en muchos otros procesos como catalizadores industriales (Levard et al. 2012; Park et al. 2012).

Los métodos de síntesis de nanoestructuras pueden clasificarse de acuerdo a la forma en que se alcance la escala nanométrica en: (1) aproximación de arriba hacia abajo (top- 
down) y (2) aproximación de abajo hacia arriba (bottom-up). Además, dependiendo de la naturaleza del proceso que se lleve a cabo pueden ser divididos en métodos químicos y métodos físicos (Hullmann 2006; Tomellini et al. 2004; Mohanraj et al. 2006). En los primeros se utilizan sales como precursores, agentes reductores, oxidantes, estabilizantes, y precursores de crecimiento preferencial (alguna molécula polimérica funcional o un surfactante catiónico, anionico o neutro). La reducción química vía húmeda, la titulación ácida y la síntesis hidrotermal son métodos de síntesis química de NPs que se caracterizan por producir altos volúmenes de producto en poco tiempo, con altos rendimientos $y$ costos relativamente bajos. Por otro lado, en los métodos físicos, en la mayoría de los casos, se parte del mismo material que se desea obtener y se utiliza un factor físico como ente promotor del crecimiento o de la disminución de tamaño. Entre las alternativas de síntesis de NPs por métodos físicos podemos destacar: deposición por vapor química, deposición de capa atómica, ablación laser, reducción radiolítica, descarga de arco eléctrico, entre otras (Hullmann 2006; Tomellini et al. 2004; Mohanraj et al. 2006).

Dentro de las aplicaciones con mayor impacto y más prometedoras de la nanotecnología se pueden citar: almacenamiento, producción y conversión de energía, armamento y sistemas de defensa, producción agrícola, tratamiento y remediación de aguas, diagnóstico de enfermedades, sistemas de administración de fármacos, procesamiento de alimentos, remediación de la contaminación atmosférica, construcción, monitorización de la salud, detección y control de plagas, control de desnutrición en lugares pobres, informática y alimentos transgénicos (Hullmann 2006; Frejo et al. 2011; Levard et al. 2012; Park et al. 2012; Sánchez et al. 2006; Zuluaga et al. 2007; Barrere et al. 2011).

\section{Aplicaciones de la nanotecnología a la agricultura y producción de alimentos.}

La nanotecnología permite el desarrollo de nuevos materiales, procesos y dispositivos que tienen una amplia gama de aplicaciones en muchos campos. Entre estos campos están la agricultura y la producción de alimentos. En primer lugar es importante indicar que, aunque la agricultura sea un mecanismo de producción de alimentos no todas las actividades de producción agrícola son de carácter alimentario. Así mismo, por producción de alimento no debe entenderse solo la parte comestible dentro de la línea de producción, sino, que en este concepto se está incluyendo el desarrollo de empaques, aditivos y cualquier otro sistema material que se encuentre en contacto con los alimentos (OMS y FAO 2011; Molins 2008; Scenihr 2006).

De acuerdo con el reciente informe conjunto entre la Organización Mundial de la Salud (OMS) y la Organización de las Naciones Unidas para los Alimentos y Agricultura (FAO, Food and Agriculture Organization), emitido en el 2011, la industria y la ciencia han reconocido el potencial de la nanotecnología en la producción de alimentos y en el sector agropecuario. Sin embargo, debido a los conocimientos limitados que se tienen de los efectos de estas aplicaciones sobre la salud humana, las partes interesadas reconocen la necesidad de considerar oportunamente las consecuencias de la tecnología para garantizar 
Palencia et al. - Toxicidad de nanopartículas sobre los microorganismos del suelo

la inocuidad de los alimentos (OMS y FAO 2011; Maynard y Warheit 2011).

La nanotecnología proyecta el desarrollo de nuevas estrategias para la obtención de productos así como aplicaciones innovadoras para la agricultura y la ganadería, el tratamiento de las aguas y la producción, elaboración, conservación y envasado de los alimentos. Actualmente, en algunos países ya se comercializan alimentos de base nanotecnológica y en muchos otros se aplica la nanotecnología en materiales para el embalaje de alimentos. Además, otros productos se hallan en fase de investigación y desarrollo o en una etapa próxima de incursión al mercado. Los productos nanotecnológicos se utilizan en una gran variedad de aplicaciones, como el envasado para evitar el deterioro de los alimentos por acción de los microorganismos, como aditivos para modificar propiedades organolépticas como la textura y el sabor, y en la fabricación de agroquímicos para la fabricación de nuevas formas de administración de plaguicidas, fertilizantes y estimulantes del crecimiento vegetal (Hullmann 2006; Frejo et al. 2011; Levard et al. 2012; Park et al. 2012).

A la vista de los grandes progresos en la agricultura y la producción de alimentos, cabe prever que en los próximos años los productos alimenticios derivados de la nanotecnología estarán cada vez más al alcance de los consumidores en todo el mundo. Además, la incorporación intencional o accidental, o si se prefiere directa o indirecta, de productos nanotecnológicos al medio ambiente será una realidad que impactará a los diferentes ecosistemas naturales y agroecosistemas (OMS y FAO 2011).

\section{NANOPARTICULAS METÁLICAS}

Las NPs metálicas son aquellas cuya naturaleza química está definida principalmente por la presencia de un metal. En sentido estricto este tipo de materiales no son en un $100 \%$ de naturaleza metálica ya que con frecuencia se requiere el uso de agentes de estabilización o, para otorgarle alguna propiedad en particular, la superficie del metal suele funcionalizarse con alguna sustancia de naturaleza orgánica (Ju-Nam y Lead 2008). A manera de ejemplo se hará énfasis en dos tipos de NPs metálicas más estudiadas y versátiles: AgNPs y $\mathrm{TiO}_{2} \mathrm{NPs}$.

\section{Nanopartículas de plata (AgNPs).}

Las AgNPs son partículas de plata cerovalente (número de oxidación cero, $\mathrm{Ag} 0$ ) obtenidas comúnmente por reducción química de una sal de plata (por ejemplo, $\mathrm{AgNO}_{3}$ ) en presencia de un agente reductor (por ejemplo, citrato de sodio) y un agente de estabilización (por ejemplo, citrato de sodio, poli(vinilpirrolidona), span 80, entre otros). Aunque la plata (Ag) ha sido utilizada por sus propiedades antimicrobianas por cientos de años, dentro de la gama de sustancias cuya actividad bactericida se está investigando, las AgNPs surgen como un prometedor agente antibacteriano que podría ser utilizado para combatir diferentes tipos de bacterias resistentes a los antibióticos. Las AgNPs han sido usadas principalmente como agentes bactericidas en diferentes productos en los cuales estás pueden producir toxicidad inducida a través de la combinación del efecto tanto de las AgNPs como de los iones plata $\left(\mathrm{Ag}^{+}\right)$que pueden producirse por la disolución de estás (Ju-Nam y Lead 2008; Moore 2006; Frejo et al. 2011).

En diversos estudios las propiedades antimicrobianas de las AgNPs se han reportado. 
Además, se ha determinado que estas tienen efecto sobre bacterias Gram negativas como Escherichia coli, Vibrio cholera, y Pseudomonas aeruginosa y sobre bacterias Gram positivas como Bacillus subtilis, Staphylococcus aureus y Enterococcus faecalis (Ju-Nam y Lead 2008; Frejo et al. 2011; Handy et al. 2008).

\section{Nanotubos de óxido de titanio ( $\left.\mathrm{TiO}_{2} \mathrm{NTs}\right)$.}

El dióxido de titanio $\left(\mathrm{TiO}_{2}\right)$ es muy utilizado para distintas aplicaciones, como por ejemplo en pigmentos para pinturas o cosméticos, como fotocatalizador o como material para celdas fotovoltaicas, entre otras. El $\mathrm{TiO}_{2}$ posee diferentes fases cristalinas, entre ellas la anatasa y el rutilo. Por otro lado, los $\mathrm{TiO}_{2} \mathrm{NTS}$ son un tipo especial de NPs de $\mathrm{TiO}_{2}$ con forma tubular, estos pueden ser obtenidos por diferentes rutas de síntesis (reducción por titulación ácida de isopropóxido de titanio o la síntesis hidrotermal una sal de titanio) (Handy et al. 2008; Levard et al. 2012). La toxicidad de las $\mathrm{TiO}_{2} \mathrm{NPs}$ ha sido estudiada frente a diversos organismos como Vibrio fischeri (bacteria luminiscente), E. coli, S. ceverise y B. subtilis. Los resultados muestran que $\mathrm{TiO}_{2} \mathrm{NPs}$ pueden influenciar la expresión genética en bacterias recombinantes, afectar diferentes cromóforos y producir cambios en el flujo metabólico de los microorganismos analizados (Bang et al. 2011; Jiang et al. 2011; Park et al. 2012).

\section{Impacto de las NPs sobre los microorganismos.}

La célula microbiana puede ser considerada como un sistema micrométrico nanoestructurado, ya que sus componentes encajan en el concepto de nanosistema, y por consiguiente, la misma naturaleza ha alcanzado un alto grado de miniaturización funcional mediante el diseño de bio-nanoestructuras. Debido a que la célula en sí, es una sistema biológico donde ocurren un sin número de procesos bioquímicos que implican una alta sincronicidad, especificidad y reactividad química, es claro que una sustancia capaz de alterar cualquiera de los componentes implicados en los procesos celulares puede ser considerada altamente dañina para la conservación de la vida del microorganismo (Handy et al. 2008).

En el suelo, los microorganismos juegan un rol muy importante en el mantenimiento de sus funciones como ecosistema y están muy relacionados con su uso para la producción agrícola. Las NPs pueden ser introducidas en los suelos como resultados de un amplio número de actividades humanas como la liberación controlada mediante diferentes tecnologías aplicadas a las aguas y los suelos (por ejemplo, fertilizantes). Además, la liberación no intencional de NPs al aire, aguas y suelos puede ocurrir mediante diversos procesos tecnológicos (por ejemplo, la deposición de residuos y la producción de subproductos dentro de un determinado proceso), o por situación no previstas (derrame de material nanoparticulado como resultado de un accidente). Por lo tanto, existen diferentes rutas por las cuales las NPs pueden ingresar a diferentes ecosistemas naturales, y sin embargo, sus propiedades ecotoxicológicas y riesgos aún no han sido completamente caracterizadas. Por ejemplo, la toxicidad microbiana para ambientes acuáticos se ha reportado para $\mathrm{TiO}_{2} \mathrm{NTs}$, fullerenos, AgNPs, NPs de $\mathrm{ZnO}$ (ZnONPs) y NPs de Au (AuNPs) (Shah et al. 2009; Nowacs 2009; Somasundaran et al. 2010; Fang 2009). 
Los efectos de las NPs sobre los microorganismos dependen de varios factores, entre ellos, de sus propiedades fisicoquímicas, de la dosis, el tiempo de contacto, del tipo de microorganismo, de las condiciones locales, y de la composición del medio de crecimiento que en algunas ocasiones pueden interactuar en sinergia para dañar o lisar las células microbianas (Gutarowska et al. 2012; Zhang et al. 2011).

En los últimos años el número de publicaciones enfocadas hacia la nanotoxicología medioambiental han incrementado, sin embargo siguen siendo pocas en comparación con el número de investigaciones en las que se buscan nuevas y novedosas aplicaciones nanotecnológicas. Por otro lado, aunque la mayoría de los estudios nanotoxicológicos han concluido que la citotoxicidad es producida a dosis muy altas de exposición, estas investigaciones destacan que las alteraciones celulares pueden tener lugar a muy bajas concentraciones de material nanoestructurado. Entre los efectos tóxicos de las NPs sobre las células se incluye la producción de especies de oxígeno reactivas de forma intracelular, la producción de estrés oxidativo, lesiones del ADN, desnaturalización de biomacromoléculas y envolvimiento a través de la superficie de la membrana (Shah et al. 2009; Nowacs 2009; Somasundaran et al. 2010; Fang 2009; Gutarowska et al. 2012).

Entre los nanomateriales que han mostrado genotoxicidad están las AuNPs, AgNPs, (Cr-Co)NPs, CoNPs, $\mathrm{TiO}_{2} \mathrm{NTs}$, ZnONPs, $\mathrm{Fe}_{2} \mathrm{O}_{3} \mathrm{NPs}$, fullerenos, entre otros. Para el caso de las AgNPs, se ha identificado que estas pueden producir estrés oxidativo y alteración de los mecanismos de transporte a través de la membrana, mientras que para $\mathrm{TiO}_{2} \mathrm{NTs}$, además de la producción de estrés oxidativo y fotoactividad, se ha indicado que estas pueden producir la muerte celular y fibrilación debido a la interferencia de las funciones de las membranas de células macrófagas (Handy et al. 2008; Shah et al. 2009; Nowacs 2009).

En el caso de las NPs coloidales los microorganismos carecen de un mecanismo de adaptación a este tipo de sustancias. Tomando como ejemplo las AgNPs, es posible encontrar reportes que evidencian que su mecanismo en las células de los microorganismos es multidireccional; es decir, ésta inhibe la replicación del ADN, perturba el potencial eléctrico y el funcionamiento de las membranas citoplasmáticas, que conduce a la salida de muchos metabolitos de las células, causa la pérdida de bioactividad de aminoácidos y altera los procesos respiratorios (Gutarowska et al. 2012; Zhang et al. 2011; Wen-Ru et al. 2010).

\section{INTERACCIÓN DE LAS NPS Y BACTERIAS A NIVEL MOLECULAR}

Una sola célula tiene un rango de tamaño de decenas de micras, lo que resulta en un enorme tamaño en comparación con una $\mathrm{NP}$ de $10 \mathrm{~nm}$. Así mismo, en comparación con una NP de $10 \mathrm{~nm}$, una molécula de un fármaco es extremadamente pequeña, lo que podría llevar a la conclusión de que una NPs es menos activa que un fármaco debido a la diferencia de tamaños. Sin embargo, la realidad es que una NP inyectada en un sistema vivo puede dar lugar a un incontable número de interacciones con dicho sistema independientemente de su tamaño (Saptarshi et al. 2013). Como consecuencia del pequeño tamaño de las NPs, existe una alta probabilidad de que estas puedan penetrar hacia el interior 
de las células y trasladarse a través de barreras de membrana (Saptarshi et al. 2013; Dawson et al. 2009; Oberdörster 2004). Así, las NPs con un tamaño menor a los $100 \mathrm{~nm}$ de diámetro se considera que pueden entrar en las células, aquellas con menos de $40 \mathrm{~nm}$ pueden entrar fácilmente en el núcleo celular y con un tamaño inferior a los $35 \mathrm{~nm}$ podrían cruzar barreras biológicas cruciales para la vida de un organismo sin que estas resulten para nada efectivas (Saptarshi et al. 2013). En el interior de la célula y de sus diversos compartimentos, las NPs pueden interactuar con biomoléculas como las proteínas, los ácidos nucleicos, lípidos y otros metabolitos debido a su pequeño tamaño y a su enorme relación masa superficie (Saptarshi et al. 2013; Dawson et al. 2009; Oberdörster 2004).

\section{Interacción de las nanopartículas metálicas con proteínas.}

Las proteínas son polipéptidos con una conformación definida y llevan una carga superficial neta que depende del $\mathrm{pH}$ del medio. Las proteínas poseen diferentes grados de ordenamiento estructural que dan lugar a diferentes "estructuras"(primaria, secundaria, terciaria y cuaternaria). Estás definen, respectivamente, la secuencia de aminoácidos a lo largo de la cadena, las formaciones tipo $\alpha$-helice o $\beta$-laminas entre otras, la formación de dominios proteicos (conformación tridimensional) y la asociación con segmentos de aminoácidos, cofactores y grupos prostéticos para formar entidades supramoleculares. Estas estructuras son posibles mediante diferentes interacciones moleculares como son los puentes de hidrógeno, interacciones electrostáticas, fuerzas de van der Waals, interacciones hidrofóbicas (que direcciona el plegamiento proteico) y enlaces covalentes (puentes disulfuros). La alteración de cualquiera de estos niveles estructurales puede generar la perdida de funcionalidad de la proteína y en consecuencia producir daños significativos en la integridad celular (Devlin 2004).

La adsorción de estas en la nanobiointerfase es promovida por varias fuerzas como los enlaces de hidrógeno, las fuerzas de solvatación y las fuerzas de Van der Waals. En el caso de las proteínas, estas pueden adsorberse en la superficie de las NPs y alterar sus diferentes formas de organización estructural. De este modo, la superficie de la NP puede modificar la estructura de las proteínas y por lo tanto su función biológica se verá afectada. Además, la superficie de las NPs también puede introducir inestabilidad termodinámica de la molécula de proteína adsorbida por lo que esta sería susceptible de desnaturalización química.

Se ha reportado que ZnONPs inducen despliegue del dominio periplásmico de la proteína ToxR de Vibrio cholera haciendo que la proteína susceptible a la desnaturalización por agentes caotrópicos (sustancias que desorganizan la estructura tridimensional en macromoléculas tales que proteínas, ADN o ARN y las desnaturaliza) (Saptarshi et al. 2013; Bardhan et al. 2009; Chatterjee et al. 2010; Gondikas et al. 2012).

En primer lugar, dado que los aminoácidos son las unidades constitutivas de las proteínas, para entender las posibles interacciones con estas resulta apropiado analizar las interacciones de las NPs con sus unidades constitutivas de manera individual. Dependiendo de la naturaleza de cada nanopartícula la interacción química resultante puede ser diferente, así que, de forma ilustrativa se ha seleccionado 
las AgNPs para mostrar este aspecto. Así, la interacción de AgNPs con unidades de cisteína, mediante la interacción covalente de la cadena de tiol con el núcleo de la nanopartículas se ha reportado en publicaciones especializadas y se sugiere que una interacción de naturaleza covalente, similar a la formación de sulfuros de plata tiene lugar (Gondikas et al. 2012; Mansoob et al. 2012; Mukherjee et al. 2012; Řezanka et al. 2011).

Por otro lado, para la síntesis de AgNPs se emplea comúnmente agentes estabilizantes. La naturaleza de la interacción entre estos agentes y la superficie de la AgNP sugiere que moléculas estructuralmente similares puedan también adsorberse superficialmente en la NP. Por ejemplo, mediante el método de Turkevich, las AgNPs pueden ser estabilizadas con citrato, una molécula con tres grupos carboxilatos de igual naturaleza a los presentes en los aminoácidos. Debido a que en las proteínas, dependiendo de los aminoácidos presentes, los grupos caboxilatos están haciendo parte de enlaces peptídicos, los aminoácidos con cadenas laterales con estos grupos son más susceptibles de promover la interacción superficial con las nanopartículas (por ejemplo, el aspartato y el glutamato) (Devlin 2004; Bardhan et al. 2009; Chatterjee et al. 2010). Además, el citrato, dependiendo del $\mathrm{pH}$ del medio, puede encontrarse protonado $(\mathrm{pH}<\mathrm{pKa}$, comúnmente en medios ácidos) o desprotonado ( $\mathrm{pH}>\mathrm{pKa}$, comúnmente en medios ligeramente ácidos, neutros o alcalinos). De este modo, el citrato estabilizaría las AgNPs mediante repulsiones electrostáticas, y estas mismas repulsiones minimizarían su interacción con grupo carboxilatos desprotonados, sin embargo, se favorecería la interacción con grupos cargados positivamente en las cadenas laterales de los aminoácidos (por ejemplo, lisina o arginina) (Gondikas et al. 2012; Řezanka et al. 2011; Railsback et al. 2012).

Las NPs también pueden afectar a las estructuras secundarias de las proteínas, y en algunos casos, causar cambios irreversibles. Las $\mathrm{TiO}_{2} \mathrm{NPs}$ han mostrado la capacidad de causar cambios conformacionales y reducir la polimerización de tubulina, que es un elemento esencial de la proteína del citoesqueleto. El efecto sobre las proteínas puede ocasionar una serie de cambios que conllevarían a la alteración del ADN, las interacciones proteína-proteína y anomalías en los procesos de reconocimiento molecular necesario para la realización de múltiples funciones metabólicas. Además, la pérdida de la actividad de las enzimas puede producirse como resultado de los cambios conformacionales en los sitios activos asociados a la actividad catalítica.

Por otro lado, las propiedades químicas de las proteínas individuales y su flexibilidad estructural también juegan un papel importante en la regulación de tales modificaciones promovidas a nivel superficial sobre las estructuras secundarias de las proteínas (Saptarshi et al. 2013; Worrall et al. 2006; Lacerda et al. 2009; Gheshlaghi et al. 2008). La composición de las NPs, su hidrofobicidad, la presencia grupos funcionales específicos, el $\mathrm{pH}$ y la temperatura han sido identificados como los principales factores que afectan a la adsorción de proteínas en la superficie de las NPs. Además, la sedimentación de estas, especialmente en experimentos in vitro se ha asociado para ser un factor importante en las de las NPs a nivel celular (Turci et al. 2010; Cho et al. 2011; Sohaebuddin et al. 2010; Gojova 
et al. 2007; Kopac et al. 2008). Por otro lado, las soluciones coloidales de NPs a menudo tienen una tendencia a formar aglomerados. El tamaño de NPs, la concentración y la carga superficial pueden influir en la formación de aglomerados. De este modo, NPs con mayor tamaño pueden más fácilmente aglomerarse y ejercer un efecto apreciablemente diferente sobre las funciones biológicas de las proteínas en comparación con las NPs que se dispersan de manera eficiente (Saptarshi et al. 2013; Herzog et al. 2009; Skebo et al. 2007).

Una de las características físicas de la NPs con mayor influencia sobre las proteínas es el tamaño. Por ejemplo, AuNPs con un tamaño de $12 \mathrm{~nm}$ se han reportado para presentar una mayor afinidad de unión con el fibrinógeno, que es una proteína soluble del plasma sanguíneo precursor de la fibrina, en comparación con AuNPs de $7 \mathrm{~nm}$. En otro estudio, las nanopartículas de sílice $\left(\mathrm{SiO}_{2} \mathrm{NPs}\right)$ con $15 \mathrm{~nm}$ inducen un cambio seis veces mayor en la estructura secundaria de proteína de la anhidrasa carbónica en comparación con $\mathrm{SiO}_{2} \mathrm{NPs}$ de $6 \mathrm{~nm}$. La anhidrasa carbónica es una enzima que pertenece a una familia de metaloenzimas y que catalizan la conversión rápida de dióxido de carbono y agua a bicarbonato y protones. Así mismo, se ha reportado un efecto de las $\mathrm{SiO}_{2} \mathrm{NPs}$ dependiente del tamaño sobre la estabilidad de la ribonucleasa A y lisozimas. Cabe aclarar que, debido al efecto de tantos factores, se puede concluir que es muy difícil formular reglas sobre las interacciones de proteínas con NPs que sean generalizables a tipos diferentes de NPs (Saptarshi et al. 2013; Lundqvist et al. 2004; Shang et al. 2007; Shi-Qiang et al. 2008).
Interacción de las nanopartículas metálicas con ácidos nucleicos.

Debido a su pequeño tamaño las nanopartículas pueden atravesar los sistemas membranosos de las células e ingresar al núcleo, ya allí estas pueden afectar de manera directa el ADN. Además, estas pueden acumularse en el citoplasma, y durante la mitosis interaccionar con el material genético de la célula. Alternativamente, los daños al ADN pueden ocurrir a través de mecanismos indirectos en los cuales las nanopartículas no interaccionan físicamente con la molécula de ADN. Es así que las nanopartículas pueden producir respuestas celulares que inducen la genotoxicidad (por ejemplo: estrés oxidativos o inflamación). Por otro lado, la inflamación es un importante proceso fisiológico en respuesta a la lesión de tejidos y es mediada por células inflamatorias que segregan una gran variedad de factores solubles, factores de inhibición y especies de nitrógeno reactivo. Estos factores pueden promover daños en el ADN en forma de fragmentación cromosomal, mutaciones puntuales y formación de aductos de ADN, adicionalmente inhiben la reparación del ADN e inducen reacciones de metilación (Gheshlaghi et al. 2008; Turci et al. 2010; Shi-Qiang et al. 2008; Shanzhou et al. 2014; Glibitskiy et al. 2012).

A partir de experimentos in vitro, se ha reportado que las NPs pueden inhibir su síntesis con un efecto dependiente de la concentración siendo este mucho mayor que el observado en partículas de la misma naturaleza pero a escala micrométrica. El mecanismo molecular de la inhibición se ha sugerido que es mediante la adsorción física de la ADN polimerasa o la adsorción química del cebador sobre la 
superficie de las NPs siendo el impacto mayor para el cebador que para la ADN polimerasa. Este fenómeno de adsorción se ve reflejado en la pérdida de funcionalidad de la biomolécula y por ende afecta todos los procesos celulares asociados con ellas. Los resultados experimentales sugieren que la adsorción química ocurre mediante la interacción de los grupos $\mathrm{P}=\mathrm{O}$ y $\mathrm{C}-\mathrm{O}-\mathrm{P}$. Por otro lado, la adsorción física ha sido relacionada mediante las diferentes interacciones que pueden surgir entre la biomolécula y la superficie de la NPs, siendo esta interacción de tipo electrostática o en algunos casos de naturaleza hidrofóbica (en ausencia de cargas). Investigaciones sugieren que estas interacciones pueden conllevar a la apertura de la doble hélice de la molécula de ADN (Sohaebuddin et al. 2010; Shi-Qiang et al. 2008; Shanzhou et al. 2014).

Recientemente, el ADN ha sido punto de atención de muchos estudios relacionados con nanotecnología debido a sus potenciales aplicaciones en la terapia génica y en otros tratamientos médicos. Se ha reportado que de manera controlada es posible la interacción entre el ADN y NPs a través de enlaces moleculares específicos y no específicos. Así, el ADN puede ser enlazado de forma covalente (unión específica) a la superficie de las NPs a través de grupos funcionales, tales como $\mathrm{OH},-\mathrm{NH}_{2} \mathrm{o}-\mathrm{COOH}$. Por ejemplo, AuNPs o AgNPs pueden ser funcionalizadas mediante oligonucleótidos con grupos $-\mathrm{NH}_{2}$. Por otro lado, la unión no específica entre el ADN y las NPs también puede alcanzarse por adsorción simple a través de interacciones no covalentes (Shi-Qiang et al. 2008).

Es importante indicar que, entre las NPs más estudiadas en relación a su interacción con el ADN, están las AuNPs y las AgNPs. En relación a las AuNPs, se cree que las fuerzas electrostáticas entre las hebras de ADN anionicas y las superficies de carga negativa de AuNPs estabilizadas con citrato son menos favorables para la unión con la doble hélice que aquellas estabilizadas mediante la formación de cargas positivas. Por otra parte, el estudio sobre la afinidad de unión de desoxinucleósidos a AuNPs reveló que los cuatro desoxinucleósidos muestran altas afinidades, mientras que la timina interactúa mucho más débilmente con la superficie de oro que otras nucleobases. Además, se ha sugerido que la rigidez de las cadenas de ADN y el tamaño de las NPs puede desempeñar un rol importante en las interacciones de los ácidos nucleicos con las AuNPs (Shanzhou et al. 2014; Glibitskiy et al. 2012; An y Jin 2012; Basu et al. 2008).

En el caso de las AgNPs, en experimentos empleando ADN genómico bacteriano se concluyó que, debido a la densidad de carga negativa en la superficie de las AgNPs estabilizadas por citrato, la interacción electrostática con grupos fosfatos de la cadena de ADN es poco probable. Ante esto, se ha sugerido más bien una interacción mediante la coordinación del metal para la formación de un complejo ADN-AgNPs. Las investigaciones muestran que estas NPs pueden unirse a las bases nitrogenadas mediante la coordinación con los átomos de nitrógeno presentes en la guanina, la adenina, la timina y la citosina (Shi-Qiang et al. 2008; Shanzhou et al. 2014; Glibitskiy et al. 2012).

Como se mostró previamente las nanopartículas metálicas pueden interaccionar a diferentes 
niveles con las proteínas, por tal razón no es de extrañar que los procesos en los que estas estén presentes se vean fuertemente afectados. En el caso de la replicación del ADN bacteriano, este procede de forma bidireccional a partir del cromosoma circular. Se comienza a partir de un punto de origen central y procede a lo largo de todo el cromosoma hasta que dos enzimas de polimerasa se juntan. En este proceso participan enzimas del tipo polimerasa y topoisomerasa. Además, otro aspecto importante durante la replicación del ADN bacteriano es el proceso de metilación. La metilación del ADN consiste en la adición de un grupo metilo en la posición 5 del anillo de pirimidin citosina o el número 6 del nitrógeno del anillo de purin adenina. Así, durante la replicación algunos de los nucleotidos a lo largo de la nueva hebra y en secciones específicas tienen incorporado grupos metilo.

Este proceso es necesario por varias razones: (1) secuencias metiladas sirven para bloquear o activar la transcripción de ARN mensajero y así actuar como un mecanismo de control para la expresión genética, (2) estas secuencias pueden servir como iniciadores para la replicación de $A D N$, (3) se ha sugerido que puede estar asociada a la reparación del ADN (Devlin 2004). Por consiguiente, durante la replicación del ADN bacteriano también estarán presentes proteínas estabilizadoras, helicasas, nucleotidos trifosfatos, polimerasas, metilasa de citosina, de adenina o metiltransferasas entre otras biomoleculas, y en consecuencia, la presencia de nanopartículas puede mediante la interacción con cualquiera de estas generar un efecto indirecto sobre la replicación del ADN.

\section{Interacción de las nanopartículas con}

fosfolípidos y membranas lipídicas.

Las NPs son altamente eficaces para penetrar la membrana plasmática y para alterar los procesos naturales dentro de la célula. Estas sirven como excelentes portadores de sustancias bioactivas a través de la membrana, probablemente debido a los mecanismos de internalización como ruptura física, el transporte de mediado a través de la membrana, la formación de poros, etc. Las membranas bacterianas poseen una composición bastante compleja y varía dependiendo de si es Gram positiva o Gram negativa (Devlin 2004).

Estudios recientes, sobre los efectos de diferentes NPs metálicas sobre la estabilidad y/o la deformación de la membrana, han revelado que la incorporación de las NPs metálicas dentro de la membrana altera el comportamiento de la fase lipídica por la disminución de la temperatura de transición de fase y el aumento de la fluidez de la bicapa. Debido a que el comportamiento de la fase lipídica influye en diferentes procesos de las membranas, la interacción de las NPs con las bicapas lipídicas se ha convertido en un muy importante tema de estudio con el fin de establecer el efecto su interacción. Algunas investigaciones relacionadas con esta interacción se han realizado con bicapas homogéneas que consisten en fosfolípidos zwitteriónicos y vesículas lipídicas (Santhosh et al. 2012; Roiter et al. 2008).

En los experimentos realizados con vesículas de lípidos, la encapsulación de las NPs puede lograrse atrapando las partículas dentro del núcleo acuoso o en la bicapa hidrofóbica. Posteriormente, para ser incluidas en la bicapa lipídica, las NPs deben poseer dos características importantes. En primer lugar, las 
NPs deben ser de menor tamaño para caber dentro de una bicapa y además deben tener una superficie hidrófobica. Cuando las NPs están atrapadas dentro de las bicapas, puede dar lugar a cambios en el empaquetamiento de los lípidos y de este modo alterar las interacciones lípido-lípido. Además, la alteración de estas interacciones interlipídicas puede resultar en cambios en el comportamiento de fase de la bicapa lipídica, que se relaciona con el grado de ordenamiento de lípidos y la viscosidad bicapa (Santhosh et al. 2012; Roiter et al. 2008; Lin et al. 2010; Wang et al. 2012).

Cuando algunas proteínas o nanopartículas cargadas se adsorben en la superficie celular, la membrana se somete a deformación y los lípidos en las bicapas constituyentes se reorganizan debido a la interacción electrostática entre los lípidos, nanopartículas y proteínas de la membrana. Puesto que la membrana está cargada negativamente, las NPs cargadas positivamente son atraídas más hacia la superficie de la membrana celular y muestran niveles más altos de internalización en comparación con partículas sin carga y con carga negativa. Por lo tanto, dependiendo de su tamaño y química superficial, las NPs incrustadas en la membrana pueden influir en la estabilidad y la función de las vesículas, la formación de dominios, la separación de fases, etc. (Roiter et al. 2008; Lin et al. 2010).

Dependiendo del tamaño, carga electrostática e hidrofobicidad, las NPs pueden quedar parcial o totalmente atrapada en la bicapa. Tres diferentes posibilidades de atrapamiento de NPs se han reportado. La primera posibilidad es el incrustamiento parcial de la NP en la bicapa, la segunda posibilidad consiste en la extensión por contacto a lo largo de la región hidrófoba y el tercer modo es el atrapamiento de NPs perfectamente en el centro de la bicapa debido a las interacciones de tipo hidrofóbico (Shanzhou et al. 2014; Glibitskiy et al. 2012; An y Jin 2012; Santhosh et al. 2012; Roiter et al. 2008; Lin et al. 2010; Wang et al. 2012).

\section{CONCLUSIONES}

Con el fin de lograr establecer el efecto de las NPs metálicas sobre los sistemas moleculares de los microorganismos del suelo con importancia agrícola mayores estudios son requeridos. Además, el efecto del ambiente químico debería ser considerado para establecer el impacto real de los potenciales productos nanotecnológicos de uso agrícola. La información consultada sugiere que el efecto de las NPs metálicas sobre los microorganismos del suelo obedece a mecanismos inespecíficos y multidireccionados, resultantes de la múltiple interacción de estás con diferentes biomoléculas. Por lo tanto, la actividad biocida que se ha reportado para un grupo determinado de bacterias podría ser fácilmente extrapolada a otras. Sin embargo, existe evidencia que la superposición de factores, tanto de las NPs como de las biomoléculas, puede generar respuestas diferentes que deberían ser consideradas y sopesadas.

\section{REFERENCIAS}

An, H. and Jin, B. 2012. Prospects of nanoparticle-DNA binding and its implications in medical biotechnology, Biotechnology Advances; 30:1721-1732

Bang, S., Lee, T., Lee, S., Kim, P. and Kim, 
J. 2011. Toxicity assessment of titanium (IV) oxide nanoparticles using Daphnia magna (Water Flea), Environmental health and toxicology; 26:c2011002

Bardhan, M., Mandal, G. y Ganguly, T. 2009. Steady state, time resolved, and circular dichroism spectroscopic studies to reveal the nature of interactions of zinc oxide nanoparticles with transport protein bovine serum albumin and to monitor the possible protein conformational changes. Journal of Applied Physic; 106:034701

Barrere, R., D'Onofrio, M., Matas, L. y Marcotrigiao, G. 2011. La Nanotecnología en Iberoamérica, situación actual y tendencias. Observatorio Iberoamericano de Ciencia, Tecnología e Innovación del Centro de Altos Estudios Universitarios de la OEI

Basu, S., Jana, S., Pande, S. y Pal, T. 2008. Interaction of DNA bases with silver nanoparticles: Assembly quantified through SPRS and SERS. Journal of Colloid and Interface Science; 321:288293

Chatterjee, T., Chakraborti, S., Joshi, P., Singh, S., Gupta, V. y Chakrabarti P. 2010. The effect of zinc oxide nanoparticles on the structure of the periplasmic domain of the Vibrio cholerae ToxR protein. Febs Journal; 277:4184-4194

Cho, E., Zhang, Q. y Xia, Y. 2011. The effect of sedimentation and diffusion on cellular uptake of gold nanoparticles.
Nat Nano; 6:385-391

Dawson, K., Salvati, A. y Lynch, I. 2009. Nanotoxicology: Nanoparticles reconstruct lipids. Nat Nano; 4:84-85

Devlin, T. 2004. BIOQUÍMICA: APLICACIONES CLÍNICAS. Cuarta edición. Reverte S. A. USA

Ezanka, P., Záruba, K. y Král, V. 2011. Supramolecular chirality of cysteine modified silver nanoparticles. Colloids and surface A: Physicochemical and Engineering Aspects, 374:77-83

Fang, J., Shan, X., Wen, B., Lin, J. y Owens, G. 2009. Stability of titania nanoparticles in soil suspensions and transport in saturated homogeneous soil columns, Environmental Pollution; 157:1101-1109

Frejo, M. T., Díaz, M. J., Lobo, M., García, J. y Capó, M. 2011. Nanotoxicología ambiental: retos actuales, Medicina Balear; 26: 36-46

Gondikas, A., Morris, A., Reinsch, B., Marinakos, S., Lowry, G. y HsuKim, H. 2012. Cysteine-Induced Modifications of Zero-valent Silver Nanomaterials: Implications for Particle Surface Chemistry, Aggregation, Dissolution, and Silver Speciation. Environmental Science \& Techonology; 46(13):7037-7045

Gheshlaghi, Z., Riazi, G., Ahmadian, S., Ghafari, M. y Mahinpour, R. 2008. Toxicity and interaction of titanium 
Palencia et al. - Toxicidad de nanopartículas sobre los microorganismos del suelo

dioxide nanoparticles with microtubule protein. Acta Biochimical Biophysical Sin (Shangai); (40):777-782

Glibitskiy, G., Jelali, V., Semenov, M., Roshal, A., Glibitskiy, D. and Yu O. 2012. Interaction of DNA with silver nanoparticles. Ukranian Journal of Physic; 57( 7):695-699

Gojova, A., Guo, B., Kota, R., Rutledge, J., Kennedy, I. and Barakat, A. 2007. Induction of inflammation in vascular endothelial cells by metal oxide nanoparticles: Effect of particle composition. Environmental Health Perspective; 115:403-409

Gutarowska, B., Skora, J., Zduniak, K. and Rembisz, D. 2012. Analysis of the sensitivity of microorganisms contaminating museums and archives to silver nanoparticles. Int. Biodeterior. Biodegrad; 68: 7-17

Handy, R., Von der Kammer, F., Lead, J., Hassellov, M., Owen, R. and Crane, M. 2008. The ecotoxicology and chemistry of manufactured nanoparticles. Ecotoxicology; 17: 287-314

Herzog, E., Byrne, H., Davoren, M., Casey, A., Duschl, A. and Oostingh, G. 2009. Dispersion medium modulates oxidative stress response of human lung epithelial cells upon exposure to carbon nanomaterial samples. Toxicology Applied Pharmacology; 236:276-281

Hullmann, A. 2006. The economic development of nanotechnology - An indicator based analysis, European Commission, Unit "Nano S\&T Convergent Science and Technologies, Alemania

IUPAC. 1995. Recommended terminology for the description of carbon as a solid. PAC, 1995, 67, 473. http://goldbook.iupac. org/G02684.html

Jiang, G., Shen, Z., Bao, Y., Chen, J. and He, T. 2011. Toxicological assessment of $\mathrm{TiO} 2$ nanoparticles by recombinant Escherichia coli bacteria. J. Environemtal monitoring; 13:42-28

Ju-Nam, Y. and Lead, R. 2008. Manufactured nanoparticles: An overview of their chemistry, interactions and potential environmental implications. Science of the total environment; 396-414

Kopac, T., Bozgeyik, K. and Yener, J. 2008. Effect of $\mathrm{pH}$ and temperature on the adsorption of bovine serum albumin onto titanium dioxide. Colloids Surface A Physicochemical Engineering Aspects; 322:19-28

Lacerda, S.; Park, J., Meuse, C., Pristinski, D., Becker, M., Karim, A. and Douglas J. 2009. Interaction of Gold Nanoparticles with Common Human Blood Proteins. ACS Nano; 4:365-379

Levard, C., Matt, E., Lowry, G. and Brown, G. 2012. Environmental Transformations of Silver Nanoparticles: Impact on Stability and Toxicity. Environmetanl Science \& Technology; 46: 6900- 6914 
Lin, J., Zhang, H., Chen, Z. and Zheng, Y. 2010. Penetration of Lipid Membranes by Gold Nanoparticles: Insights into Cellular Uptake, Cytotoxicity, and Their Relationship. ACS Nano; 4(9):5421-5429

Lundqvist, M., Sethson, I. and Jonsson, B. 2004. Protein Adsorption onto Silica Nanoparticles. Conformational Changes Depend on the Particles' Curvature and the Protein Stability. Langmuir; 20:10639-10647

Mansoob, M., Kalathil, S., Lee, J. and Hwan, M. 2012. Synthesis of Cysteine Capped Silver Nanoparticles by Electrochemically Active Biofilm and their Antibacterial Activities. Bulletin Korean Chemical Society 22:2592-2596.

Maynard, A. and Warheit, M. 2011. The New Toxicology of Sophisticated Materials: Nanotoxicology and Beyond, Toxicological Sciences; 120: 109-129

Mohanraj V. and Chen Y. 2006. Nanoparticles- A review, Tropical Journal of Pharmaceutical Research; 59: 561-573

Molins, R. 2008. Oportunidades y amenazas de la nanotecnología para la salud, los alimentos, la agricultura y el ambiente, Comunica-Perspectivas, Innovación y Tecnología; 38-53.

Moore, M. 2006. Do nanoparticles present ecotoxicological risks for the health of the aquatic environment?. Environment International; 32: 967-976
Mukherjee, S., Menegazzo, N., Brooksh, K., Dhurjati, P., Smorodin, V. y Nohe, A. 2012. Synthesis of L-Cysteine Stabilized Silver Nanoparticles and Their Effects on Cell Viability. Advanced Science Letters; 6:26-33

Nowacs, B. 2009. The behavoior and effects of nanoparticles in the environment. Environmental pollution; 1 (57):10631064

Oberdörster, G., Sharp, Z., Atudorei, V., Elder, A., Gelein, R., Kreyling, W. and Cox C. 2004. Translocation of Inhaled Ultrafine Particles to the Brain. Inhalation Toxicology; 16:437-445

OMS y FAO. 2011. Informe Reunión conjunta $\mathrm{FAO} / \mathrm{OMS}$ de expertos acerca de la aplicación de la nanotecnología en los sectores alimentario y agropecuario: posibles consecuencias para la inocuidad de los alimentos. Roma

Park, S., Lee, S., Lee, J., Sim, S., Gu, M., Yi J. and Lee, J. 2012. Toxic effects of titanium dioxide nanoparticles on microbial activity and metabolic flux, Biotechnology and bioprocess engineering; 17:276-282

Railsback, J., Singh, A., Pearce, R., McKnight, T., Collazo, R., Sitar, Z., Yingling, Y. and Melechko, A. 2012. Weakly Charged Cationic Nanoparticles Induce DNA Bending and Strand Separation. Advanced Materials; 24:4261-4265 
Roiter, Y., Ornatska, M., Rammohan, A, Balakrishnan, J., Hein, D. and Minko,

S. 2008. Interaction of Nanoparticles with Lipid Membrane. Nanoletters 8 (3) 941-944.

Sánchez, J., Guerrero, H., Marín, S., Tamayo, R. y Cosme, M. 2006. Nano: nanotecnología en España, Comunidad de Madrid

Santhosh, P., Penic, S., Genova, J., Iglic, A., Kralj-Iglic, V. and UIrih, N. 2012. A study on the interaction of nanoparticles with lipid membranes and their influence on membrane fluidity. Journal of Physics. Conference series 2012; (398): 012034

Saptarshi, S., Duschl, A. and Lopata, A. 2013. Interaction of nanoparticles with proteins: relation to bio-reactivity of the nanoparticle. Journal of Nanobiotechnology; 11(26): 1-12

Scientific committee on emerging and newly identified health risks (SCENIHR). 2006. The appropriateness of existing methodologies to assess the potential risks associated with engineered and adventitious products of nanotechnologies. European Commission for Health and Consumer, Bruselas

Shah, V. and Belozerova, I. 2009. Influence of Metal Nanoparticles on the Soil Microbial Community and Germination of Lettuce Seeds. Water air pollunt. 197:143-148
Shang, W., Nuffer, J., Dordick, J. and Siegel, R. 2007. Unfolding of Ribonuclease A on Silica Nanoparticle Surfaces. Nano Letter; 7:1991-1995

Shanzhou, H., Peng, Liu. and Yu H. 2014. Aggregation of Gold Nanoparticles and DNA Damage by Atomic Force Microscopy, Journal of Wuhan University of Technology-Material Science Education; 29(1):180-184

Shi-Qiang, L., Hong, Z., Rong-Rong, Z., XiaoYu, S., Si-De, Y. and Shi-Long, W. 2008. Impact and mechanism of TiO2 nanoparticles on DNA synthesis in vitro. Science in China Series B: Chemistry; 51:367-372

Skebo, J., Grabinski, C., Schrand, A., Schlager, J. and Hussain, S. 2007. Assessment of metal nanoparticle agglomeration, uptake, and interaction using high-illuminating system. International Journal of Toxicology;26:135-141

Sohaebuddin, S., Thevenot, P., Baker, D., Eaton, J. and Tang, L. 2010. Nanomaterial cytotoxicity is composition, size, and cell type dependent. Particle and Fibre Toxicology; 7: 22.

Somasundaran, P., Fang, X., Ponnurangam, S. and Li, B. 2010. Nanoparticles: Characteristics, mechanims and modulation of biotoxicity. KONA powder and particle journal; 28:38-49

Tomellini, R. and Hullmann, A. 2006. 
Nanotechnology - Innnovation for tomorrow's world. DirectorateGeneral for research, Nanoscience and nanotechnologies, Alemania, 2004

Turci, F., Ghibaudi, E., Colonna, M., Boscolo, B., Fenoglio, I. and Fubibi, B. 2010. An Integrated Approach to the Study of the Interaction between Proteins and Nanoparticles. Langmuir; 26:83368346

Wang, T., Bai, J., Jiang, X. and Ulrich, G. 2012. Cellular Uptake of Nanoparticles by Membrane Penetration: A Study Combining Confocal Microscopy with FTIR Spectroelectrochemistry. ACS Nano; 6(2):1251-1259

Wen-Ru, L., Xiao-Bao, X., Qing-Shan, S., Hai-Yan, Z., You-Sheng, O-Y. and YiBe, C. 2010. Antibacterial activity and mechanism of silver nanoparticles on Escherichia coli. Applied Micobiology Biotechnology; (85):1115-1122
Worrall, J., Verma, A., Yan, H. and Rotello, V. 2006. "Cleaning" of nanoparticle inhibitors via proteolysis of adsorbed proteins. Chemical Communication; 2338-2340

Zhang, X., Niu, H., Yan, J. and Cai, Y. 2011. Immobilizing silver nanoparticles onto the surface of magnetic silica composite to prepare magnetic disinfectant with enhanced stability and antibacterial activity. Colloids and Surface; 375:186192

Zuluaga, D., Sánchez, J., Aguilera, A. and Medina, J. 2007. Métodos de Fabricación de Nanotecnología. Informe de vigilancia tecnológica. Colciencias, Bogotá 